\title{
PESSOAS, PROCESSOS ETECNOLOGIA NA GESTÃO DO CONHECIMENTO: UMA REVISÃO DA LITERATURA
}

\section{People, Processes and Technology in Knowledge Management: A Literature Review}

\section{Maria Lúcia Corrêa Neves}

Doutoranda em Engenharia e Gestão do Conhecimento na Universidade Federal de Santa Catarina. Florianópolis, SC. Brasil. e-mail:lucia.c.neves@uol.com.br

\section{Gregório jean Varvakis}

Doutor em Manufacturing Engineering - Loughborough University of Technology. Professor titular da Universidade Federal de Santa Catarina, Departamentoto de Engenharia do Conhecimento.

Florianópolis, SC. Brasil.e-mail: g.varvakis@ufsc.br

\section{Francisco Antonio Pereira Fialho}

Doutor em Engenharia de Produção, e Engenharia do Conhecimento. Professor Titular da Universidade Federal de Santa Catarina. Florianópolis, SC. Brasil.e-mail:fapfialho@gmail.com

\section{RESUMO}

Esta pesquisa qualitativa foi realizada com o objetivo de analisar a abordagem dos elementos "pessoas, processos e tecnologia" nos estudos acadêmicos de Gestão do conhecimento (GC). A partir de uma revisão integrativa da literatura, foram analisados estudos relacionados na base de dados Scopus, identificados por abordarem, concomitantemente, GC e os três elementos. Partiu-se do pressuposto que a presença dos elementos "pessoas, processos e tecnologia", identificada em publicações oriundas de GC e, também, em distintas áreas de pesquisas, sinaliza a sinergia interdisciplinar necessária para a abordagem das complexas questões organizacionais. A pesquisa identificou um conjunto de 70 publicações contendo, concomitantemente, GC e os três elementos. Utilizando diversos filtros, foi identificado um subconjunto de nove publicações para leitura integral e síntese. A análise das publicações permitiu identificar três diferentes categorias de estudos abordando a relação entre os construtos. A pesquisa permitiu as seguintes considerações: (1) os três elementos são, recorrentemente, mencionados nos estudos de GC, sem o aprofundamento que contribuiria para a sinergia entres os distintos saberes que caracterizam os processos de gestão organizacional; e (2) o amadurecimento e consolidação de GC como disciplina, permanecem dependentes da superação da abordagem fragmentada das questões organizacionais.

Palavras-chave: Gestão do conhecimento. Pessoas, processos e tecnologia. Gestão.

\section{ABSTRACT}

This qualitative study aimed to analyze the "people, processes and technology" factors approach in academic studies of knowledge management (KM). After an integrative review of literature drawn from the international database Scopus, relevant scientific studies were analyzed and identified per their approach, namely, KM and the three factors, simultaneously. The "people, processes and technology" factors identified in various KM publications and in studies of different research areas point to interdisciplinary synergy as being necessary for approaching such complex organizational issues. Thus, this study identified a set of 70 publications containing KM and the three factors, simultaneously. After applying various filters, a second set of nine publications were identified for thorough reading. In the analysis of publications, three distinct study categories regarding the relationship of these constructs were identified. Aside from category synthesis, this study allowed for the following considerations: (1) the three factors are recurrently mentioned in KM studies, but without the necessary depth that would contribute towards a synergy among the distinct principles that characterize organizational management processes; (2) advancement and consolidation of KM as a discipline depends on subjugating the fragmented approach to organizational issues.

Keywords: knowledge management; people, processes and technology; interdisciplinary; management. 


\section{INTRODUÇÃO}

A gestão do conhecimento (GC) é definida como um conjunto de processos que orienta a criação, disseminação e utilização do conhecimento, para atingir os objetivos da organização (DAVENPORT; PRUSAK, 1998).

As teorias e práticas de GC emergiram na década de 90, como uma abordagem estratégica capaz de assegurar a sustentabilidade organizacional na sociedade do conhecimento e permanecem se destacando entre as estratégias corporativas mais discutidas na atualidade (INKINEN, 2016).

Os estudos acadêmicos seminais sobre GC e, também, publicações não acadêmicas, recorrentemente, abordam a relação entre GC e a tríade formada por pessoas, processos e tecnologia. Os três elementos são apresentados, alternadamente, como pilares, elementos centrais ou dimensões de GC, algumas vezes, acompanhados de outro elemento, por exemplo, estrutura organizacional, cultura ou liderança.

Considera-se, neste estudo, que a menção à necessidade de equilíbrio da configuração dos três elementos está relacionada com a imprescindibilidade de sinergia entre saberes diversos, para tratar dos complexos problemas experimentados pelas organizações no século XXI.

Nesta direção, foi desenvolvida esta pesquisa que teve como objetivo identificar se a abordagem dos elementos pessoas, processos e tecnologia nos estudos seminais de GC contribuiu para que teorias e práticas da disciplina avançassem na direção da integração e sinergia interdisciplinar.

Para tanto, foi realizada a revisão integrativa dirigida à base multidisciplinar Scopus para identificar, analisar e apresentar, de forma sintetizada, as abordagens dos três elementos nos estudos de GC. A pesquisa qualitativa é apresentada neste artigo, que está estruturado por uma fundamentação teórica, seguida de procedimentos metodológicos; análise e discussão dos resultados da revisão integrativa da literatura e conclusões, com possíveis direcionamentos para pesquisas futuras.

\section{FUNDAMENTAÇÃO TEÓRICA}

A fundamentação teórica é apresentada a seguir, considerando-se GC em relação aos pilares: pessoas, processos e tecnologia, também, as questões de multidisciplinaridade e interdisciplinaridade e, ainda, o elemento pessoas com relação ao trabalho e ao desempenho de processos e tecnologia.

\subsection{Gestão do conhecimento e a relação com os pilares pessoas, processos e tecnologia}

Os estudos de gestão, tradicionalmente, utilizaram conhecimentos oriundos da matemática, economia e engenharia, como saberes adequados para tratar dos ativos tangíveis ou capital financeiro, expressos por dados quantitativos e de origem econômica, típicos do século XX (KAPLAN; NORTON, 2004).

Bygrave (1989) observa que, ao longo do século $\mathrm{XX}$, muitos dos teóricos de administração eram engenheiros, sendo que o vínculo oriundo da formação em engenharia implicou em tentativas de criar uma abordagem estruturada e racional das organizações.

No final do século XX, no entanto, foi evidenciado que o valor das organizações não estava relacionado, apenas, ao valor dos ativos tangíveis típicos da sociedade industrial (EDVINSSON; MALONE, 1998). Gradativamente, percebeu-se a participação e, até mesmo, a predominância dos ativos intangíveis, denominados de ativos intelectuais, capital intelectual ou simplesmente conhecimento (EDVINSSON;MALONE, 1998).

A partir desta constatação, o conhecimento e o processo de aprendizado que renova o conhecimento, antes assuntos de interesse da área gestão de recursos humanos ou gestão de pessoas, passaram a ser pauta estratégica, originando teorias e práticas da disciplina GC (PRAHALAD; HAMEL, 2001).

Os ativos que integram o capital intelectual, por sua natureza, são de base qualitativa e subjetiva, desafiando os teóricos de GC, frequentemente, influenciados pelas temáticas das disciplinas objetivas (KAPLAN; NORTON, 2004).

Existem muitas definições para GC. Para Dalkir (2013), é a coordenação intencional e sistemática do conhecimento das pessoas de uma organização, da 
tecnologia, dos processos e estrutura organizacional, a fim de agregar valor, através da reutilização e inovação do conhecimento gerido.

Dalkir (2013) destaca que a definição proposta no parágrafo anterior permite a visão multidisciplinar da GC, como abordagem de gestão organizacional. Os elementos pessoas, processos e tecnologia estão contemplados na definição sinalizando a necessidade de saberes diversos para o entendimento e o tratamento das questões organizacionais.

É importante destacar que as abordagens de gestão organizacional que antecederam a GC receberam críticas pela visão disciplinar. Por exemplo, Fischer (2002, p. 24) alertou que Henry Ford, nos seus estudos, abordava os fatores humanos como recursos de produção. Este mesmo autor destaca que o teórico de estratégia Michael Porter, em seu livro sobre estratégia competitiva (PORTER, 2004), dedicou "não mais de duas páginas" para tratar de práticas de gestão de pessoas. Esta fragilidade é observada, também, em teorias e práticas de reengenharia propostas por Hammer e Champy (2009). Para Fischer (2002, p.24), as publicações sobre o modelo de gestão reengenharia focaram exclusivamente os processos, ignorando a dimensão humana, que aparece "pouco ou quase nada" nos estudos sobre este modelo de gestão.

Servin e De Brun (2005, p. 46) consideram que é amplamente aceita, a ideia de que a GC precisa ser pensada em termos dos três elementos. Os autores ainda recomendam que, para iniciativas de GC bem-sucedidas, seja considerada a influência da cultura, dos valores e dos comportamentos, como característica do elemento pessoas, além da necessidade de buscar processos apropriados e tecnologias certas.

Para Servin e De Brun (2005), muitos pesquisadores comparam os três componentes à pilares de sustentação da GC, destacando que a ausência de reflexão e ação sobre um pilar, compromete os resultados de GC. Os três elementos podem ser entendidos, também, como facilitadores ou barreiras ao uso efetivo das práticas de GC e, ainda, como tópicos que devem ser contemplados em um plano de implantação de GC. O pilar referente ao elemento pessoas é mais importante do que os outros, tendo em vista tratar-se do elemento criador do conhecimento.

A visão de Servin e De Brun (2005) sobre cada elemento é apresentada no quadro 01.

Quadro 01 Elementos básicos de GC

\begin{tabular}{|c|c|c|}
\hline PESSOAS & PROCESSOS & TECNOLOGIA \\
\hline $\begin{array}{l}\text { Dos três elementos é o mais importante, } \\
\text { tendo em vista o conhecimento ser algo que } \\
\text { emana das pessoas: elas criam, comparti- } \\
\text { Iham e usam o conhecimento. A influência } \\
\text { da cultura, dos valores e dos comportamen- } \\
\text { tos integra o elemento Pessoas. }\end{array}$ & $\begin{array}{l}\text { O elemento envolve dois aspectos: (1) os } \\
\text { processos de GC que suportam, junto com } \\
\text { os outros elementos, a implantação de GC e } \\
\text { cujas ações são direcionadas para a infraes- } \\
\text { trutura que sustenta o ciclo GC; (2) o olhar } \\
\text { estratégico para os processos organizacio- } \\
\text { nais e a infraestrutura que geram barreiras ou } \\
\text { facilitam o ciclo GC. }\end{array}$ & $\begin{array}{l}\text { O elemento é um importante facilitador da } \\
\text { maioria de iniciativas de GC. Atua em duas } \\
\text { formas principais: (1) fornecendo os meios } \\
\text { para as pessoas organizarem, armazenarem } \\
\text { e acessarem o conhecimento explícito e a } \\
\text { informação; (2) Ajudando a conectar pessoas } \\
\text { com pessoas, viabilizando o compartilha- } \\
\text { mento do conhecimento tácito. }\end{array}$ \\
\hline
\end{tabular}

Fonte: Servin e De Brun (2005) adaptado pelos autores

Servin e De Brun (2005) destacam que processos e as tecnologias são importantes por que agregam valor à organização, através da redução de custos, tempo e esforços das pessoas, facilitando que estas compartilhem conhecimento e informações necessários para atingimento dos objetivos de GC. Em relação ao elemento tecnologia, Servin e De Brun (2005) destacam que este agrega valor quando reduz o custo, tempo e esforços necessários para as pessoas compartilharem conhecimento e informação, facilitando a aplicação e uso do conhecimento. Os autores afirmam, no entanto, que o investimento em tecnologia, ainda que de excelente qualidade, não agregará valor para a organização se: (a) sobrecarregar as pessoas de informação; (b) for desalinhado das necessidades organizacionais gerando tarefas percebidas como adicionais e não facilitadoras das tarefas e atividades de rotina; e (c) estar em desacordo com os modos de trabalhar das pessoas (SERVIN; DE BRUN, 2005).Alguns autores consideram, como 
pilares de GC, além da tríade, um ou mais elementos. Por exemplo, Nair e Prakash (2009) consideram que a GC estuda a liderança, além das pessoas, os processos e as tecnologias. Para alguns teóricos, no entanto, liderança é um processo (NORTHOUSE, 2017) e, assim, está contemplada no elemento processos, que reúne fluxos físicos e virtuais, criando barreiras ou contribuindo para o ciclo de GC.

Há ainda autores que incluem a cultura como um pilar da GC. Mas, neste estudo, adota-se a visão de Servin e De Brun (2005) que, como outros, contemplam cultura, comportamento organizacional e individual como temas abrangidos pelo elemento pessoas, assim como liderança é um tema abrangido pelo elemento processos.

\subsection{A multidisciplinaridade e interdisciplinaridade da GC}

Autores como Vergara e Branco (1993), no final do século passado, alertaram que, como disciplina consolidada, o futuro de GC dependia da superação da fragmentação em gestão (VERGARA; BRANCO, 1993).

Siegle et al. (2014, p.255) afirmam que a disciplina GC demanda "contribuições individuais do saber" e, principalmente, "integração e sinergia interdisciplinar".

Cafezeiro et al. (2016), também, destacam o papel da interdisciplinaridade, sinalizando a impossibilidade dos "saberes ditos exatos" ou dos "saberes ditos humanos" resolverem, "por si só", os desafios experimentados pela ciência e tecnologia contemporâneas (CAFEZEIRO et al., p. 110-111).

Considera-se, neste artigo, que a superação da fragmentação em gestão pode ser expressa, de forma simplificada, com abordagem e diálogo entre os saberes oriundos das disciplinas relacionadas com pessoas, processos e tecnologia (saberes ditos exatos e humanos), e, especialmente, pela reflexão sobre os efeitos do elemento pessoas e suas subjetividades nas iniciativas e resultados das teorias e práticas de GC.

Para Dalkir (2013), uma das poucas áreas de consenso nos estudos de GC, é a sua multidisciplinaridade. A autora considera que GC envolve o conhecimento de: (1) ciência organizacional; (2) ciência cognitiva; (3) educação e formação; (4) antropologia e sociologia; (5) tecnologias da informação; (6) tecnologias colaborativas; (7) linguística e linguística computacional; (8) informação e biblioteconomia; (9) comunicação e storytelling; (10) escrita técnica e linguística.

Dalkir (2013) dedica um capítulo do seu livro sobre GC à abordagem da influência da cultura organizacional no ciclo e nos resultados da aplicação de GC. A autora afirma ainda que a cultura é poderosamente subjetiva e reflete os significados e entendimentos que, normalmente, atribui-se às situações e às soluções que se aplicam a problemas comuns.

Bhatt (2001) afirma que a cultura e a história organizacional impactam a sinergia entre os elementos pessoas, processos e tecnologia, e, por esta razão, as estratégias de GC não podem facilmente serem replicadas por outras organizações. Para o autor, uma organização não é sistema tecnológico nem um sistema social, tratando-se de um sistema de experiências pessoais, relações sociais, processos e tecnologias. O elemento pessoas, neste caso, contempla os conhecimentos tácito e explícito das pessoas e, também, suas experiências e relações desenvolvidas no trabalho, podendo facilitar ou dificultar o ciclo que promove produtos, serviços e processos de excelência ou inovadores (BHATT, 2001).

Servin e De Brun (2005), também, consideram que os resultados de GC são dependentes da cultura organizacional e comportamental.

Schein (1999) afirma que a cultura organizacional é um padrão de suposições descobertas ou desenvolvidas por um determinado grupo que define como ele, o grupo, lida com seus problemas de adaptação externa e integração interna. Isso é considerado válido porque funciona bem e, nesta direção, deve ser ensinado aos novos membros como a forma correta de perceber, pensar e sentir em relação a esses problemas. A posição do autor evidencia a relação da cultura com o conhecimento e, até mesmo, com a aprendizagem.

A preocupação com a questão cultural, reforça o vínculo de GC com a antropologia (DALKIR, 2013). No entanto, na visão de Mascarenhas (2002), de forma geral, os estudos de gestão, ao abordarem cultura, têm preocupações teóricas diferentes das que motivam a antropologia. Para o autor, a antropologia estuda a cultura sem a preocupação de produzir modelos 
ou ferramentas para a intervenção ou o controle, enquanto a Administração é essencialmente prática focando, como objetivo de análise, as variáveis principalmente internas, que influenciam no desempenho organizacional. Nesta direção, Mascarenhas (2002) afirma que a cultura organizacional tende a ser percebida nas organizações, apenas, como mais uma variável, cujas características devem ser controladas ou manipuladas objetivando, exclusivamente, a maximização de resultados.

Com esta visão, Jaime Junior (2002, p. 42) afirma que predomina nos estudos de gestão, a abordagem de cultura caracterizada como funcionalista, um "sistema simbólico harmônico" que, gerenciado, pode contribuir para a "maximização da performance organizacional". O autor alerta que existem, nas organizações, distintas versões de cultura organizacional, provenientes de pessoas com "diferentes capitais econômicos, culturais e simbólicos”, envolvidas não apenas no processo produtivo, mas, também, no complexo jogo político que caracteriza as organizações, indicando a necessidade de lidar com questões subjetivas (JAIME JUNIOR, 2002, p. 42).

\subsection{O elemento pessoas nos estudos de GC, em contraposição aos estudos que abordam as relações de trabalho}

Svetlik e Stavrou-Costea (2007) revisaram a literatura que explora a conexão entre a forma de gerir pessoas e GC, identificando poucos estudos que fazem essa conexão explícita. Monteiro e Cardoso (2012), por sua vez, analisando os estudos que tratam do relacionamento de GC e o elemento pessoas, afirmaram que, frequentemente, as teorias e práticas de GC são apresentadas como: (1) se fossem "tecnicamente e politicamente" neutras no contexto organizacional ou (2) imunes às influencias das suposições subjacentes e pré-existentes em relação ao valor do trabalho e aos trabalhadores.

Paralelamente, nas últimas décadas, as pesquisas das áreas que tratam das relações de trabalho relatam aumento de conflitos no ambiente organizacional de diversos países, além de uma dicotomia entre discurso e práticas de gestão (PFEFFER, 2007).

Castells (2018), na virada do milênio, já destacava a dificuldade das empresas em compreender a di- nâmica e a complexidade do ambiente organizacional e das relações de trabalho na sociedade pós-industrial. Como exemplo, o autor citava que, algumas empresas ainda estavam adaptando suas políticas internas. Mas, na dinâmica diária do trabalho, as mulheres e os representantes de outras minorias, como gays, lésbicas, bissexuais, transexuais, já reivindicavam os espaços que mereciam nas organizações.

Na visão de autores como Kowske et al. (2010) e Conway et al. (2015), a dificuldade de compreensão em relação à complexidade das organizações permanece, resultando na tensão que marca o ambiente organizacional do século XXI.

Kowske et al. (2010) citam alguns títulos de publicações não acadêmicas que tratam da tensão no ambiente organizacional, por exemplo, "Campo de batalha psicológica" e "A indigesta convivência de geração milênio com seus chefes". Os mesmos autores afirmam que, frequentemente, a análise superficial do problema aponta, como causas dos conflitos, as questões relacionadas aos valores das novas gerações no trabalho ou as diferenças entre as gerações, relacionando, indevidamente, o comportamento dos trabalhadores às fragilidades e aos conflitos percebidos.

Neves et al. (2015) consideram que os conflitos derivados dos problemas geracionais, recorrentemente alardeados pelas publicações empresariais, podem indicar a dificuldade das organizações lidarem com a diversidade em geral e não especificamente com a diversidade geracional. Os autores ponderam que muitas organizações convivem de forma conflituosa com a diversidade demográfica ou de interesses, citando conflitos com clientes, representantes de sindicatos ou órgãos reguladores.

Deal et al. (2010) abordam a tendência de gestores questionarem o grau de comprometimento dos trabalhadores com as organizações, sem a devida reflexão sobre a reciprocidade expressa pela qualidade do comprometimento das organizações com os trabalhadores. Consideram que as práticas da reengenharia provocaram e provocam, nos trabalhadores (pessoas), desconfiança e apatia em relação às empresas, refletindo no aumento de rotatividade. É perceptível, no século XXI, o rompimento com a ideia de que o trabalho é central na vida das pessoas ou que se trata de um vínculo perene, o que colabora 
para explicar a elevação no índice de rotatividade funcional (DEAL et al., 2010).

É manifesto o interesse em identificar nos estudos de GC, especialmente nos que mencionam os elementos pessoas, processos e tecnologias, a reflexão sobre os aspectos subjetivos e situacionais, que afetam as aplicações das teorias produzidas na academia, ou a confirmação de que se segue adotando uma perspectiva objetiva, para tratar das relações de trabalho (KAUFMAN, 2010).

Considera-se neste estudo que, especialmente com a falta de perspectiva histórica nos estudos de gestão e de GC, a ausência de contextualização dificulta a compressão sobre as especificidades do exercício da gestão (VIZEU, 2010). Em contrapartida, a investigação acadêmica ancorada no contexto histórico contribui para a compreensão de diferentes questões da atual realidade organizacional brasileira (SANTOS et al., 2016).

\subsection{O elemento pessoas afetando o desempenho dos elementos processos e tecnologia}

A necessidade de sinergia entre os elementos pessoas, processos e tecnologia não é uma especificidade do ciclo ou dos processos envolvidos na implantação de GC. O elemento pessoas impacta a gestão geral dos processos das organizações, afetando ainda o uso e o desempenho das novas tecnologias.

Gino e Pisano (2008), analisando a literatura relacionada com processos e operações, afirmam que, tradicionalmente, menciona-se a relevância do fator humano para o funcionamento e o desempenho de tarefas, atividades ou operações. Contudo, nos textos sobre teorias e práticas, é também característica a presunção de que as pessoas são racionais ou podem ser induzidas a apresentar comportamento racional.

Por sua vez, Croson et al. (2013) consideram que, apenas recentemente, a análise do comportamento humano nas decisões relacionadas com gestão de processos, despertou a atenção dos teóricos da área.

Siegler et al. (2014) abordam a necessidade de alinhamento entre as áreas de gestão de pessoas e gestão de processos, tanto no campo teórico como prático. As autoras citam a nova linha de pesquisa em gestão de operações, denominada de behavioral operations (operações comportamentais). A busca pelo novo conhecimento foi considerada, pelas autoras, uma demonstração de que emergiu na área, finalmente, o interesse por pesquisas que abordem os aspectos subjetivos do comportamento humano.

Na visão de Croson et al. (2013), em pesquisas sobre operações comportamentais, busca-se superar a visão simplista e mecânica do comportamento humano, com investigações que tratam das consequências e do impacto do comportamento subjetivo, nas operações das organizações.

Anteriormente, a visão simplista foi percebida por Faria e Fonseca (2014). Mais recentemente, esses autores revisaram os seus estudos organizacionais que abordam a cultura e sua relação com produtos, serviços ou processos inovadores. Assim, alertaram que, mesmo nas pesquisas envolvendo o processo de inovação, contemplando alto grau de subjetividade, ainda predomina a utilização de métodos quantitativos e a objetividade.

Abordando os elementos pessoas, processos e tecnologia, as duas publicações mais antigas identificadas na base Scopus são de 1993. Os dois estudos são sobre tecnologia da informação e foram publicados no periódico IBM Systems.

Em um dos artigos publicados, Kenn (1993) afirma que a efetiva fonte de vantagem competitiva das organizações é a capacidade de garantir a gestão integrada de pessoas, processos (de negócios) e tecnologia, ressaltando que, como recurso, a tecnologia não é diferencial, porque é acessível para todas as organizações.

Na outra publicação, há um artigo em que Griss (1993) descreve a possibilidade de reutilização sistemática de software, como estratégia para melhoria da qualidade de produtos, redução de tempo de desenvolvimento e custo de produção. Contudo, destaca que a reutilização eficaz não se restringe à tecnologia (códigos), devendo também ser direcionada para as boas práticas relacionadas à experiência das pessoas - usuários e equipes envolvidas no desenvolvimento.

Os exemplos anteriores apresentam o conteúdo de pesquisas que abordam a dimensão pessoas, tratando de tecnologia da informação. Mas, assim como as publicações que tratam de processos, a abordagem de pessoas nos estudos relacionados com tecnologia, 
também, tende a utilizar o pressuposto de que as pessoas são racionais (GINO; PISANO, 2008).

Dalkir (2013) aborda o desperdício de tempo e dinheiro em muitas organizações que estabeleceram seus programas de GC, a partir da adoção de tecnologia da informação. Por exemplo, investindo no desenvolvimento de um portal web ou adotando qualquer outra ferramenta de colaboração, com a convicção de que, a partir disso, os comportamentos envolvidos no ciclo de GC: captura, criação, compartilhamento e aplicação do conhecimento, prontamente, seriam também adotados pelas pessoas.

Com visão semelhante, Davenport e Pruzak (1998) argumentavam no século passado que, para a GC, o aumento do poder de comunicação é muito mais relevante do que o crescimento do poder de processamento.

Preocupados com a questão, autores de estudos mais recentes de GC, ao tratarem dos benefícios da tecnologia, abordam a necessidade de concomitante adoção de práticas de GC que, efetivamente, estimulem o compartilhamento, a confiança e a colaboração, entre outros aspectos subjetivos do comportamento humano (ANDREEVA; KIANTO, 2012).

Por exemplo, a integração de GC com pessoas e tecnologia é proposta por Khatri et al. (2010) que abordam as iniciativas de GC em organizações de serviços. A premissa dos autores é que pessoas e tecnologias integradas são centrais nos processos de negócios, porque oferecem excelência aos clientes e, por isso, não são apenas elementos de apoio às organizações.

\section{PROCEDIMENTOS METODOLÓGICOS}

Para o trabalho empírico, que investigou a menção de pessoas, processos e tecnologia nos estudos de GC, utilizou-se o método de revisão sistemática definida como uma revisão planejada para responder a uma questão de pesquisa específica (CASTRO, 2006).

Na revisão sistemática, adotam-se métodos explícitos e sistemáticos nos processos de identificação, seleção e avaliação dos estudos a serem revisados e também nas ações de coleta e análise do conteúdo dos estudos escolhidos para a revisão (BOTELHO et al. 2011).

Dentre os tipos de metodologias de trabalho existentes para a revisão bibliográfica sistemática, foi adotada a revisão integrativa. De acordo com Whitemore e Knafl (2005), esse tipo de revisão viabiliza a síntese dos estudos já publicados e, ao levarem-se em consideração os resultados de estudos anteriores, são gerados novos conhecimentos para o desenvolvimento da teoria. Em síntese, foi observada a sequência das etapas definida por Botelho et al. (2011) para a revisão integrativa.

A revisão da literatura foi realizada na base de dados eletrônica internacional Scopus, escolhida por permitir uma visão multidisciplinar e integrada de fontes relevantes para a pesquisa em questão. A base Scopus reúne importantes periódicos brasileiros.

A estratégia de busca adotada inicialmente não restringiu os tipos de documentos científicos nem delimitou data. A busca na base de dados Scopus foi realizada no dia 14/10/2016.

A primeira etapa da busca utilizou, como descritor, o construto "Gestão do Conhecimento" em inglês (Knowledge management). A ferramenta de busca foi direcionada para os campos "títulos, resumos e palavras chave" das publicações, resultando em $56.880 \mathrm{mil}$ publicações. Na sequência, aplicou-se um segundo filtro, utilizando o descritor "pessoa, processos e tecnologia", em todas as ordens, no singular e plural (utilização do sinal '*'), na língua inglesa ("process*, people ${ }^{*}$ and technology". "process ${ }^{*}$, technology and people*", "people process ${ }^{\star}$ and technology", "technology, people $e^{\star}$ and process*", "technology, process ${ }^{\star}$ and people*”).

Esta etapa de busca resultou em 70 publicações, pré-selecionadas por conterem, concomitantemente, os dois descritores investigados.

A segunda etapa da investigação contemplou a leitura exploratória dos resumos, além de títulos e palavras-chave das 70 publicações pré-selecionadas. Foi identificado, nesta etapa, um conjunto de 21 publicações que mencionavam os construtos sem relacioná-los. Na sequência, procedeu-se uma nova leitura integral do conjunto de 49 publicações. Não tendo sido identificadas publicações que, especificamente, tratavam de sinergia ou natureza interdisciplinar da GC, decidiu-se adotar uma categorização 
para classificar o novo subconjunto, cujas categorias são apresentadas na próxima seção.

A terceira etapa da investigação tratou da seleção final realizada com objetivo de refinar o conjunto de documentos que representariam as categorias. Foram selecionados documentos publicados a partir de 2011, contemplando uma visão recente do avanço das teorias de GC na direção da integração dos elementos pessoas, processos e tecnologia, sem caracterizar uma síntese do conhecimento científico sobre o objeto investigado, como inicialmente foi previsto.

\section{ANÁLISE E DISCUSSÃO DOS RESULTADOS DA REVISÃO INTEGRATIVA DA LITERATURA}

Nesta seção, a síntese dos achados da pesquisa é apresentada por categoria destacando-se que, no conjunto dos 70 documentos, não foram identificados estudos específicos sobre a sinergia ou a natureza interdisciplinar dos elementos de GC.

Isso pode ser observado sob duas visões: (1) primeiramente, pode-se considerar que a estratégia adotada, que associou a menção dos elementos pessoas, processos e tecnologia, como indicativo para localizar textos que tratam da sinergia ou que abordam a necessidade de superar a fragmentação de conhecimentos, não foi adequada; ou (2) pode-se considerar ainda que o tema não vem atraindo atenção de pesquisadores. Além disso, também não foram identificados estudos de GC com visão crítica.

Após o processo de análise, foram indicadas três categorias que permitem estruturar o conhecimento investigado sobre a relação entre pessoas, processos e tecnologia: (a) abordagem fragmentada; (b) diálogo superficial entre saberes; (c) saberes exatos e humanos relacionados.

A análise do conjunto permitiu considerar que são mais frequentes, os estudos de GC que mencionam os elementos com abordagem fragmentada, seguidos pelos estudos que tratam do diálogo entre os elementos de forma superficial, sendo escassos, os estudos que refletem sobre as implicações geradas pela relação entre os elementos.

\subsection{Abordagem fragmentada}

Pode-se considerar que a maior parte dos estudos de GC permanece sinalizando o elemento tecnologia, como prioridade nas implantações de GC, deixando pouco evidente a interferência de questões subjetivas na direção oposta ao alerta de Davenport e Pruzak (1998).

Como exemplo de estudo que sintetiza o conteúdo desta categoria, cita-se a pesquisa de Moscoso et al. (2016) que propõem um framework, denominado de guia prático, para empresas ou indivíduos implementarem iniciativas de GC. Os autores afirmam que o framework proposto é diferenciado dos demais modelos disponibilizados na literatura, especialmente por esses últimos incorporarem duas práticas de gestão oriundas da tecnologia: arquitetura corporativa e Business Inteligente. Os autores relacionam GC com os três elementos, afirmando que estes três pilares são fontes de conhecimento da organização. Quando os autores abordam o elemento pessoas, o destaque é para os seus conhecimentos e os mecanismos que podem ser adotados para capturá-los e transferi-los. Os autores abordam ainda a elevada taxa de turnover nos Estados Unidos (11\% em 2014), mencionando que este indicador sinaliza e reforça a necessidade de adoção de estratégias para manter e transferir conhecimentos do grupo de empregados que estão deixando as Organizações. Por fim, mencionam a média dos gastos com treinamento de funcionários nos EUA e a possibilidade de redução destes custos, a partir do estabelecimento de mecanismos para a captura de conhecimentos e adoção de sistemas de gestão da aprendizagem.

A fragmentação do conhecimento fica evidenciada no estudo de Moscoso et al. (2016), quando apenas ações relacionadas com codificação e armazenamento são apresentadas como possibilidades para retenção de conhecimentos. Assim, não é considerada a possibilidade de preservação de conhecimento, através de políticas de retenção e satisfação das pessoas no trabalho.

O estudo de Simamora (2015) considera GC uma abordagem gerencial que se destaca pela capacidade de melhorar o desempenho dos processos e, nesta direção, melhorar os serviços aos clientes. $\mathrm{O}$ autor reforça que o conhecimento é cada vez mais 
percebido como uma base para criar vantagem competitiva. A concepção do autor sobre os três elementos é evidenciada no resultado que traz a seguinte consideração: a adoção de uma nova "tecnologia" facilitou significativamente um "processo", permitindo que o desempenho dos empregados (pessoas) fosse melhor. A análise deste texto indica que os três elementos se relacionam por causa e efeito, não contribuindo para disseminação da necessária integração e alinhamento entre as pessoas, processos e tecnologia proposta por Bhatt (2001). Além disso, evidenciam o vínculo do elemento pessoas apenas com a maximização de resultados, conforme alertado por Mascarenhas (2002).

O estudo de Oliveira et al. (2014) apresenta os elementos pessoas, processos e tecnologia como categorias para classificar os diferentes mecanismos de GC existentes. Por exemplo, o mecanismo "Comunidades de prática” é identificado como um mecanismo de conversão de conhecimento correspondente ao elemento pessoas. Por sua vez, o mecanismo "lições aprendidas" é relacionado com conversão de conhecimento por processos e o mecanismo "portal" em conversão de conhecimento por tecnologia. Os autores observaram a adoção destes mecanismos por 11 empresas, classificando-os como fases:

1. Fase de "adoção", identificando as organizações que utilizam somente elementos tecnológicos para conversão de conhecimento em GC;

2. .Fase de "difusão", identificando as organizações que adotam os mecanismos classificados como tecnológicos e, também, de processo;

3. Fase de infusão, identificando as organizações onde é possível observar a presença de mecanismos de conversão classificados tanto como pessoas, como processos e tecnologias.

Isso que foi apresentado indica uma abordagem contrária à visão de Dalkir (2013), como foi descrita na fundamentação teórica, alertando sobre a possibilidade de retorno frágil das implantações focadas em mecanismos e tecnologia, sem considerar a cultura.

\subsection{Diálogo superficial entre saberes}

Foi possível identificar uma categoria de estudos com três elementos, agregando as considerações de autores apresentados na fundamentação teórica sobre: cultura, valores, experiências e avançando na direção da multidisciplinaridade.

Nas publicações que descrevem a categoria identificada, é possível observar alertas sobre a existência de questões subjetivas nas aplicações de GC. Mas, de forma geral, a temática destacada neste artigo é apenas tangenciada, confirmando a percepção de Faria e Fonseca (2014) de que a abordagem superficial e a utilização de métodos quantitativos e objetivos ocorrem mesmo nos estudos de GC que investigam temas com alto grau de subjetividade, como inovação ou compartilhamento.

O estudo de Rahmatia e Surendro (2013) destaca o aumento da concorrência e, por esta razão, a necessidade das empresas gerirem o conhecimento. Os autores evidenciam que, para o mundo dos negócios, a única fonte sustentável de vantagem competitiva é o conhecimento e que as teorias e práticas propostas pela GC viabilizam o refino do gerenciamento do conhecimento existente ou passível de ser desenvolvido nas organizações. Rahmatia e Surendro (2013) recomendam o investimento nas pessoas - seus valores e experiências, além de processos e tecnologia. Em síntese, o estudo propõe um modelo para aplicação de GC em empresas inteligentes.

No estudo de Andrawina e Kurniawati (2012) consta a menção de que GC será cada vez mais abordada com uma perspectiva interdisciplinar. Os autores definem GC como os processos de aprendizagem eficazes associados à exploração, aproveitamento e partilha de conhecimento humano (tácito e explícito), utilizando tecnologia e ambientes culturais para melhorar o capital intelectual e desempenho de uma organização. O sucesso de GC é apresentado como dependente da sincronia e integração entre os três elementos. O estudo trata, especialmente, de uma das ferramentas de GC, a comunidade de prática, assinalando como esta ferramenta colabora na solução de problemas ou na melhoria de processos.

Na visão Andrawina e Kurniawati (2012), as comunidades de prática oportunizam o processo de criação do conhecimento proposto por Nonaka. Os 
autores citam o conhecimento existente nas pessoas, nos processos e na tecnologia e o conhecimento novo agregado quando se introduz mais tecnologia. Isso implica em novos conhecimentos para as pessoas provenientes de compartilhamentos ou processos mais inteligentes.

Chaves et al. (2016) abordam questões complexas na prática de gestão de projetos contemporâneos, sinalizando a necessidade de uma abordagem mais adequada para enfrentar os desafios impostos pelos atuais projetos dinâmicos. Nesta direção, é proposto um modelo teórico em lições aprendidas, adicionando tecnologias da $W e b$ e incentivando gerentes de projetos a introduzirem tecnologias e recursos emergentes na sua rotina, como os benefícios das plataformas Twitter e WhatsApp, o uso de hashtags para facilitar a pesquisa e a divulgação, além de wikis, microblogs ou ferramentas de edição colaborativa. Os autores apresentam ainda teorias e práticas de GC, destacando o modelo SECI e a relevância do $B a$, proposto por Nonaka, para enfatizar a importância do contexto em que o conhecimento é compartilhado, criado e usado.

\subsection{Saberes exatos e humanos relacionados}

Nesta categoria de estudos, fica evidenciado a relação entre os saberes oriundos dos três elementos e a impossibilidade dos saberes disciplinares resolverem, isoladamente, os desafios experimentados pelas organizações contemporâneas, como sinaliza Cafezeiro et al. (2016).

Por exemplo, Dannemann (2011) propõe uma reflexão sobre o papel da tecnologia na GC, cujo objetivo da GC é facilitar e incentivar o intercâmbio de conhecimentos relacionados com atividades e processos que, especialmente, envolvam pessoas, processos e tecnologia. $\mathrm{O}$ autor também defende que as implantações de GC não devem iniciar pela tecnologia, evitando imposição de uma ferramenta que não seja adequada à cultura dos trabalhadores. Assim, reflete a respeito dos fatores que levam as pessoas ao entusiasmo pela comunicação ou compartilhamento de conhecimentos, ponderando que práticas externas e atrativas, como remuneração, reconhecimento ou promoção do trabalho, podem produzir efeito temporário. Dannemann (2011) defende que o fator mais bem-sucedido para iniciar uma cultura de compartilhamento de conhecimento é, simplesmente, identificar a fonte de conforto que desperta a vontade para a troca de conhecimentos e práticas. Para o autor, muitas vezes, isso é deflagrado pelo contato face-a-face e não pelo uso de tecnologias.

Wang (2011) trata o conhecimento como um recurso crítico organizacional, que gera vantagens competitivas sustentáveis. Por isso, assegura o atingimento dos objetivos de curto prazo e, também, a prosperidade das organizações no longo prazo. O autor resgata a importância de as organizações tratarem GC estrategicamente e vinculada com a missão, estratégias e as metas. Além disso, reforça a tese de que GC deve integrar o planejamento estratégico organizacional global, visando, especialmente, facilitar a criação de vantagens competitivas. $\mathrm{O}$ autor ainda relata a crise vivenciada por uma empresa que, repentinamente, sofreu um protesto público, causando insegurança nos clientes e multiplicando a demanda por contato com a organização. Assim, os empregados sobrecarregados com a demanda extra, que congestionava os canais, deixaram de cumprir as demandas tradicionais, constituindo um círculo vicioso que, cada vez mais, alimentou a crise.

De maneira didática, o caso descrito anteriormente ilustra a condição de dependência e complexidade estabelecida na interseção entre pessoas, processos e tecnologia, que é pertinente à GC. Isso é especialmente grave em situações de crise ou momentos que destoam da rotina organizacional.

Yusoff et al. (2011) são autores de um artigo publicado no periódico International Journal of Interdisciplinary Social Sciences. O escopo do periódico sinaliza a natureza interdisciplinar da abordagem. $\mathrm{O}$ trabalho empírico baseado em estudo de casos com um conglomerado local, identifica como distintos perfis de cultura corporativa podem impactam vários processos de GC. Os autores afirmam que os teóricos que tratam da implementação de GC, tradicionalmente, consideram o papel da cultura corporativa como a variável moderadora. Mas, ponderam que a cultura corporativa é a base determinante do projeto e da estratégia de GC. Os autores ainda sugerem que a estratégia de implantação de GC deve ser desenhada, observando-se o estágio atual da cultura e os 
processos já existentes. Assim, após um diagnostico, deve-se introduzir em doses estratégicas mecanismos ou ferramentas, abrangendo pessoas e aspectos do processo e da tecnologia. O processo é recomendado até um momento em que este se torne, naturalmente, incorporado na cultura da a organização.

\section{CONCLUSÕES}

Neste artigo, são apresentados os resultados de uma pesquisa, cujo objetivo foi analisar a abordagem dos elementos "pessoas, processos e tecnologia" nos estudos de gestão do conhecimento (GC).

Em parte, a pesquisa foi realizada porque teorias e práticas de gestão, oriundas das ciências sociais ou engenharias e anteriores ao desenvolvimento de $\mathrm{GC}$, receberam críticas devido às tentativas de tratar as questões organizacionais com visão disciplinar, expressa pelo foco dominante em um dos três elementos: pessoas, processos ou tecnologia.

Aqui foram citados autores que indicam como excessivo e nocivo o foco em um dos elementos citados. Por exemplo, por seu foco em processos, a abordagem Reengenharia foi indicada expressão característica da fragmentação dos estudos de gestão no século XX.

Partiu-se do pressuposto de que a recorrente menção aos elementos "pessoas, processos e tecnologia" nos estudos seminais de GC, poderia ter contribuído para que teorias e práticas deste modelo de gestão, avançassem na sinergia interdisciplinar necessária para o tratamento das complexas questões de gestão.

Em síntese, a pesquisa realizada foi a revisão integrativa da literatura e análise dos estudos científicos selecionados na base de dados internacional Scopus, sem delimitação de tipo de documento ou de data. Como resultado da revisão sistemática, identificou-se um conjunto de 70 publicações mencionando, concomitantemente, GC e os elementos pessoas, processos e tecnologia, em diferentes ordenações. Utilizando-se diversos filtros, foi identificado um subconjunto de nove publicações para leitura integral e síntese. $\mathrm{O}$ conjunto com nove publicações é apresentado no Apêndice 1.
A análise das publicações permitiu identificar três categorias que estruturam o conhecimento investigado sobre a relação entre pessoas, processos e tecnologia em GC: (a) abordagem fragmentada; (b) diálogo superficial entre saberes; e (c) saberes exatos e humanos relacionados.

Considera-se que a pesquisa atingiu o objetivo. Foram identificados, explorados e analisados os estudos que relacionam os três elementos com GC. No entanto, o conjunto identificado não confirmou o pressuposto da pesquisa, devido ao impacto reduzido do apelo interdisciplinar em GC. Isso permitiu a consideração de duas hipóteses: (1) a estratégia de pesquisa adotada não foi a mais adequada, já que as pesquisas de GC que evidenciam e refletem sobre a característica interdisciplinar da disciplina não foram identificadas na busca; ou (2) as pesquisas de GC, de forma geral, não estão avançando em direção à interdisciplinaridade, repetindo as fragilidades derivadas do tratamento de questões organizacionais com conhecimento fragmentado ou visão disciplinar.

No conjunto formado pelas 56,880 mil publicações que mencionam GC, apenas setenta abordam, nos campos "títulos, resumos e palavras chave", os elementos pessoas, processos e tecnologia, nesta ou em outra ordem, somando um número pouco significativo. Pode se considerar que os elementos pessoas, processos e tecnologia, mencionados frequentemente nos textos seminais de GC, tornaram-se menos frequentes ao longo da evolução da disciplina.

Foi possível concluir que a maioria das publicações que abordam a tríade, apresentam os elementos com tratamento que não instiga a reflexão multidisciplinar ou interdisciplinar. Mesmo as publicações de GC que contemplam temas subjetivos, também, utilizam predominantemente métodos quantitativos e objetivos, abordando superficialmente as questões relacionadas com o contexto organizacional e nacional. São escassas, as publicações que aprofundam a reflexão sobre a influência de questões ambíguas e subjetivas na aplicação e resultados de GC.

São igualmente raros os estudos de GC que aprofundam a reflexão sobre os temas presentes nos estudos críticos em administração ou nas pesquisas da área das ciências humanas. Por exemplo, temas como: o impacto da cultura local na aplicação das teorias e práticas desenvolvidas em outros contextos; 
os conflitos derivados das relações de poder; questões de gênero; questões geracionais ou a evolução da perda de vínculo com a empresa; o decréscimo da centralidade no trabalho; a desconfiança generalizada nas autoridades, e outros temas relacionados com a subjetividade no trabalho.

Paralelamente, constatou-se que as pesquisas oriundas de outras disciplinas relatam, com frequência, a dicotomia presente nos estudos organizacionais cujos discursos tratam de sustentabilidade, valorização do conhecimento ou habilidade para gerar relações construtivas. Os estudos sobre as práticas sinalizam recorrentes problemas éticos; ênfase no lucro de curto prazo; ganhos de mercado oriundos do envolvimento em esquemas de corrupção; irresponsabilidade social e ambiental; interferências políticas nos processos de seleção e de promoção na carreira, entre outros temas críticos.

A omissão das questões indicadas anteriormente nos estudos de GC pode induzir à equivocada percepção de que os resultados de GC serão atingidos, independentemente, do contexto interno ou externo às organizações.

Nesta direção, observou-se que os pesquisadores de GC tendem a, por exemplo, alertar que as pessoas podem relutar em adotar uma tecnologia de compartilhamento, prescrevendo, como solução, políticas de incentivo à troca de conhecimentos, sem questionar se o modelo mental que orienta as decisões da organização, genuinamente, valoriza a participação dos trabalhadores.

De forma geral, as práticas de GC, envolvendo pessoas, processos e tecnologia, são recomendadas, desacompanhadas de reflexão sobre o contexto histórico da organização, omitindo a interferência, por exemplo, da ausência de vivencia democrática nas experiências de participação e compartilhamento de conhecimento. Como a convivência com o processo de escravidão, colonização ou governos autoritários afetam a participação e iniciativa das pessoas nas organizações?

Pode-se considerar que a ausência da perspectiva histórica nos estudos de GC, dificulta a compressão sobre as especificidades do exercício da gestão, por exemplo, no Brasil.

Considera-se também que a consolidação da GC como disciplina, permanece demandando o tra- tamento destas questões e a construção de repertório interdisciplinar de teorias e práticas próprias, priorizando o equilíbrio e diálogo necessário entre questões objetivas e subjetivas, qualitativas e quantitativas que neste texto, foi traduzida como, no mínimo, a reflexão sobre o diálogo contextualizado entre os elementos pessoas, processos e tecnologia.

Espera-se que este estudo possa contribuir, ainda que modestamente, no processo de sensibilização dos pesquisadores, ao reforçar a importância e oportunidade da disciplina se destacar pelo exercício do intercâmbio entre áreas.

Destaca-se que este trabalho apresenta limitações relativas à escolha de uma única base de dados e à estratégia para identificar estudos de GC que, mais contundentemente, abordam a interdisciplinaridade. Pesquisas futuras podem preencher lacunas. Inclusive, identificando outra estratégia para localizar, no conjunto de publicações de GC, teóricos e teorias que se destacam por buscar superar ou minimizar a abordagem fragmentada do conhecimento. Por fim, podem ainda ser sugeridas outras pesquisas sobre o processo evolutivo de interação dos saberes em outros campos de pesquisa, como os que investigam a crise social e ambiental, identificando as lições já aprendidas e que podem ser replicadas em GC.

\section{AGRADECIMENTO}

À Coordenação de Aperfeiçoamento de Pessoal de Nível Superior (CAPES) pela bolsa de apoio ao doutorado.

\section{REFERÊNCIAS}

ANDRAWINA, L.; KURNIAWATI, A. Framework for Community of Practice based on SECI method and KM cycle. In ICT and Knowledge Engineering (ICT \& Knowledge Engineering), 2012. 10th International Conference on, p. 189-193, 2012. 
ANDREEVA, T.; KIANTO, A. Does knowledge management really matter? Linking knowledge management practices, competitiveness and economic performance. Journal of Knowledge Management, v. 16, n. 4, p. 617-636, 2012.

ARIFFIN, N. H. M.; HAMDAN, A. R.; OMAR, K.; JANOM, N. Customer Relationship Management (CRM) implementation: A soft issue in knowledge management scenario. In Humanities, Science and Engineering (CHUSER), p. 485-489, 2012.

BHATT, G. D. Knowledge management in organizations: examining the interaction between technologies, techniques, and people. Journal of Knowledge Management, v. 5, n.1, p. 68-75, 2001.

BOTELHO, L. L. R.; CUNHA, C. C. D. A.; MACEDO, M. O método da revisão integrativa nos estudos organizacionais. Gestão e Sociedade, v. 5, n. 11, p. 121-36, 2011.

BYGRAVE, WILLIAM D. The entrepreneurship paradigm (I): a philosophical look at its research methodologies. Entrepreneurship Theory and practice, v. 14, n. 1: 7-26, 1989.

CAFEZEIRO, I.; DA COSTA, L. C.; DA SILVA KUBRUSLY, R. Ciência da Computação, Ciência da Informação, Sistemas de Informação: Uma reflexão sobre o papel da informação e da interdisciplinaridade na configuração das tecnologias e das ciências. Perspectivas em Ciência da Informação, v. 21, n. 3, p. 111-133, 2016.

CASTELLS, M. O poder da identidade. São Paulo: Paz e Terra, 2018.

\section{CASTRO, A. A. Curso de revisão sistemática} e metanálise. São Paulo: LED-DIS/UNIFESP, 2006. Disponível em: <http://www.virtual.epm. br/cursos/metanalise> Acesso em: 15. Out. 2016.
CHAVES, M. S.; DE ARAÚJO, C. C. S.; TEIXEIRA, L. R.; ROSA, D. V.; JÚNIOR I. G.; NOGUEIRA, C. D. A new approach to managing Lessons Learned in PMBoK process groups: The Ballistic 2.0 Model. International Journal of Information Systems and Project Management, v. 4, n.1, p. 27-45, 2016.

CONWAY, E.; FU, N.; MONKS, K.; ALFES, K.; BAILEY, C. Demands or Resources? The relationship between practices, employee engagement, and emotional exhaustion within a hybrid model of employment relations. Human Resource Management, 2015.

CROSON, R.; SCHULTZ, K.; SIEMSEN, E.; YEO, M. L. Behavioral operations: the state of the field. Journal of Operations Management, v.31, n. 1-2, p. 1-5, 2013.

DANNEMANN, S. Can knowledge management survive without information technologies? Proceedings of the European Conference on Knowledge Management, ECKM, v. 2, p. 1103 1106, 2011.

DAVENPORT, Thomas H.; PRUSAK, Laurence. Conhecimento empresarial: como as organizações gerenciam o seu capital intelectual. Rio de Janeiro: Campus, 1998.

DALKIR, KIMIZ. Knowledge management in theory and practice. Routledge, 2013.

DEAL, J. J.; ALTMAN, D. G.; ROGELBERG, S.G. Millennials at work: What we know and what we need to do (if anything). Journal of Business and Psychology, v. 25, n. 2, p. 191-199, 2010.

\section{EDVINSSON, L.; MALONE, M. S. Intellectual capital. London: Piatkis, 1998.}

FARIA, F. B.; FONSECA, M. V. Cultura de Inovação: Conceitos e Modelos Teóricos. Revista de Administração Contemporânea, v.18, n. 4, p. $372,2014$. 
FISCHER, A. L. Um resgate conceitual e histórico dos modelos de gestão de pessoas. As pessoas na organização. São Paulo: Gente, 1, 11-34, 2002.

GINO, F.; PISANO, G. Toward a theory of behavioral operations. Manufacturing \& Service Operations Management, v. 10, n. 4, p. 676-691, 2008.

GRISS, M. L. Software reuse: From library to factory. IBM systems journal, v. 32, n.4, p. 548566, 1993.

HAMMER, Michael; CHAMPY, James. Reengineering the Corporation: Manifesto for Business Revolution, A. Zondervan, 2009.

INKINEN, H. Review of empirical research on knowledge management practices and firm performance. Journal of Knowledge Management, v. 20, n. 2, p. 230-257, 2016.

JAIME JÚNIOR, P. Um texto, múltiplas interpretações: Antropologia hermenêutica e cultura organizacional. Revista de Administração de Empresas, v. 42, n. 4, pág. 1-12, 2002.

KAPLAN, Robert S.; NORTON, David P. Mapas estratégicos: convertendo ativos intangíveis em resultados tangíveis. Gulf Professional Publishing, 2004.

KHATRI, N., BAVEJA, A., AGRAWAL, N. M., BROWN, G. D. HR and IT capabilities and complementarities in knowledge-intensive services. The International Journal of Human Resource Management, v. 21, n. 15, p. 2889-2909, 2010

KAUFMAN, B. E. The theoretical foundation of industrial relations and its implications for labor economics and human resource management. Industrial and Labor Relations Review, v. 64, n.1, p. 74108, 2010.
KEEN, P. G. W. Information technology and the management difference: a fusion map. IBM Systems Journal, v. 32, n. 1, p. 17-39, 1993.

KOWSKE, B. J.; RASCH, R.; WILEY, J. Millennials' (lack of) attitude problem: An empirical examination of generational effects on work attitudes. Journal of Business and Psychology, v. 25, n. 2, p. 265-279, 2010.

MASCARENHAS, A. Etnografia e cultura organizacional: uma contribuição da Antropologia à Administração de empresas. Revista de Administração de Empresas, v. 42, n. .2, p. 1-7, 2002.

MONTEIRO, S.; CARDOSO, L. Human resources management role in knowledge management-a structural model. In European Conference on Knowledge Management, p. 783. Academic Conferences International Limited, 2012.

MOSCOSO-ZEA, O.; LUJÁN-MORA, S.; CÁCERES, C. E.; SCHWEIMANNS, N. Knowledge Management

work using Enterprise Architecture and Business Intelligence. In 18th International Conference on Enterprise Information Systems (ICEIS), p. 244-249, 2016.

NAIR, P.; PRAKASH, K. Knowledge management facilitator's guide. Asian Organization Productivity, 2009.

NEVES, M.L.; FIALHO, F.; PERASSI, R. Evitando o estereótipo da cultura geracional na percepção do comportamento social organizacional. Anais do VIII Congresso Internacional Roa Bastos. Universidade Federal de Santa Catarina, p. 148-162, 2015. Disponível em $<$ https://drive.google.com/file/d/0B69YK9VlbzREXzNjZldmbWEyeW8/view>. Acesso em 14 dez. 2016.

NORTHOUSE, P. G. Leadership: Theory and practice. California: Sage Publications, 2017. 
OLIVEIRA, M.; MAÇADA, A. C. G.; CURADO, C. Adopting knowledge management mechanisms: Evidence from Portuguese organizations. Knowledge and Process Management, v. 21, n. 4, p. 231-245, 2014.

PRAHALAD, C. K.; HAMEL, GARY. The core competence of the corporation. Harvard Business Review, 2001.

PFEFFER, J. Human Resources from in Organizational Behavior Perspective: Some Paradoxes Explained. Journal of Economic Perspectives, v. 21, n. 4, p. 115-134, 2007.

PORTER, Michael E. Estratégia competitiva: técnicas para análise de indústrias e da concorrência. Elsevier, 2004.

RAHMATIA, D.; SURENDRO, K. Knowledge management system design model for smart enterprise. In ICT for Smart Society (ICISS), 2013. International Conference, p. 1-6. IEEE, 2013.

SANTOS, J. V. P., DEPÁDUA CARRIERI, A., PEREIRA, V. F., \& MARTINS, T. S. Pesquisa histórica em administração: a (re) construção identitária da Galeria do Ouvidor em Belo Horizonte (MG). Revista de Ciências da Administração, v. 18, n. 46, 2016.

SCHEIN, E.H., The corporate culture survival guide, V. 158. John Wiley \& Sons. 1999.

SERVIN, Géraud; DE BRUN, C. ABC of Knowledge Management. NHS National Library for Health, Specialist Libray, july 2005.

SIEGLER, J., BIAZZIN, C., FERNANDES, A. R. Fragmentação do conhecimento científico em administração: uma análise crítica. Revista de Administração de Empresas, v. 54, n. 3, p. 254, 2014.

SIMAMORA, B. H. The impact of request tracker application as knowledge management system on employee performance. Journal of Theoretical and Applied Information Technology, v.76, n. 2, 2015.
SVETLIK, I., STAVROU-COSTEA, E. Connecting human resources management and knowledge management. International Journal of Manpower, v. 28, n 3/4, p. 197-206, 2007.

VERGARA, Sylvia. C., BRANCO, Paulo. D. Em busca da visão de totalidade. Revista de administração de empresas, v. 3, n.6, p. 20-31, 1993.

VIZEU, F. Potencialidades da análise histórica nos estudos organizacionais brasileiros. Revista de Administração de Empresas, v. 50, n. 1, p. 37-47, 2010.

WANG, W. T. Examining the use of knowledge management during issue management. Management Research Review, v. 34, n. 4, p. 436-449, 2011.

WHITEMORE, R., KNAFL, K. The integrative review: update methodology. J Adv Nurs, v. 52, n.5, p. 546-53, 2005.

YUSOFF, W. F. W., OMAR, S. A. Knowledge Management Strategy and Design: The Role of Corporate Culture. International Journal of Interdisciplinary Social Sciences, v. 6, n. 2, 2011. 


\section{APÊNDICE 1 - CONJUNTO DE DOCUMENTOS DE GC} SELECIONADOS PARA REVISÃO INTEGRAL E SÍNTESE.

\begin{tabular}{|c|c|c|c|}
\hline AUTOR & TíTULO & PERIÓDICO & ANO \\
\hline Dannemann, S. & $\begin{array}{l}\text { Can knowledge management survive without infor- } \\
\text { mation technologies? }\end{array}$ & $\begin{array}{l}\text { Proceedings of the European Conferen- } \\
\text { ce on Knowledge Management }\end{array}$ & 2011 \\
\hline Yusoff, W.F.W., Omar, S.A. & $\begin{array}{l}\text { Knowledge management strategy and design: The } \\
\text { role of corporate culture }\end{array}$ & $\begin{array}{l}\text { International Journal of Interdisciplinary } \\
\text { Social Sciences }\end{array}$ & 2011 \\
\hline Wang, W.-T. & $\begin{array}{l}\text { Examining the use of knowledge management } \\
\text { during issue management }\end{array}$ & Management Research Review & 2011 \\
\hline $\begin{array}{l}\text { Ariffin, N.H.M., Hamdan, A.R., } \\
\text { Omar, K., Janom, N. }\end{array}$ & $\begin{array}{l}\text { Customer Relationship Management (CRM) imple- } \\
\text { mentation: A soft issue in knowledge management } \\
\text { scenario }\end{array}$ & In Humanities, Science and Engineering & 2012 \\
\hline Andrawina, L., Kurniawati, A. & $\begin{array}{l}\text { Framework for Community of Practice based on SECI } \\
\text { method and KM cycle }\end{array}$ & ICT \& Knowledge Engineering & 2012 \\
\hline Rahmatia, D., Surendro, K. & $\begin{array}{l}\text { Knowledge management system design model for } \\
\text { smart enterprise }\end{array}$ & ICT for Smart Society & 2013 \\
\hline $\begin{array}{l}\text { Oliveira, M., Maçada, A.C.G., } \\
\text { Curado, C. }\end{array}$ & $\begin{array}{l}\text { Adopting Knowledge Management Mechanisms: } \\
\text { Evidence from Portuguese Organizations }\end{array}$ & Knowledge and Process Management & 2014 \\
\hline Simamora, B.H. & $\begin{array}{l}\text { The impact of request tracker application as knowled- } \\
\text { ge management system on employee performance }\end{array}$ & $\begin{array}{l}\text { Journal of Theoretical and Applied Infor- } \\
\text { mation Technology }\end{array}$ & 2015 \\
\hline $\begin{array}{l}\text { Moscoso-Zea, O., Luján-Mora, S., } \\
\text { Cáceres, C.E., Schweimanns, N. }\end{array}$ & $\begin{array}{l}\text { Knowledge management framework using enterprise } \\
\text { architecture and business intelligence }\end{array}$ & $\begin{array}{l}\text { In 18th International Conference on } \\
\text { Enterprise Information Systems (ICEIS) }\end{array}$ & 2016 \\
\hline $\begin{array}{l}\text { Chaves, M.S., de Araújo, C.C.S., } \\
\text { Teixeira, L.R., Rosa, D.V., Glória J. } \\
\text { Junior, Nogueira, C.D. }\end{array}$ & $\begin{array}{l}\text { A new approach to managing lessons learned in } \\
\text { PMBoK process groups: The ballistic } 2.0 \text { model }\end{array}$ & $\begin{array}{l}\text { International Journal of Information } \\
\text { Systems and Project Management }\end{array}$ & 2016 \\
\hline
\end{tabular}

Fonte: base Scopus consultada em outubro de 2016. 